\title{
Correction to: Does increased femoral antetorsion predispose to cartilage lesions of the patellofemoral joint?
}

\author{
Johannes Oppermann ${ }^{1} \cdot$ Jan Bredow ${ }^{1} \cdot$ Boris Wissussek $^{2} \cdot$ Christian Karl Spies $^{3} \cdot$ Christoph Kolja Boese $^{1}$. \\ Shi-Min Chang ${ }^{4} \cdot$ Peer Eysel $^{1}$. Jens Dargel ${ }^{1}$
}

Published online: 31 March 2018

(c) European Society of Sports Traumatology, Knee Surgery, Arthroscopy (ESSKA) 2018

\section{Correction to: \\ Knee Surg Sports Traumatol Arthrosc \\ (2017) 25:2695-2701 \\ https://doi.org/10.1007/s00167-015-3625-5}

In the original article, one of the co-author's (Boris Wissussek) family name has been published incorrectly. The correct family name should be Wissussek.
The original article can be found online at https://doi.org/10.1007/ s00167-015-3625-5.

Johannes Oppermann

johannes.oppermann@uk-koeln.de

Jan Bredow

jan.bredow@uk-koeln.de

Boris Wissussek

boris-alexander.wissussek@cellitinnen.de

Christian Karl Spies

dr.christian.spies@vulpiusklinik.de

Christoph Kolja Boese

christoph.boese@uk-koeln.de

Shi-Min Chang

shiminchang11@aliyun.com

Peer Eysel

peer.eysel@uk-koeln.de
Jens Dargel

jens.dargel@uk-koeln.deje

1 Department of Orthopedics and Trauma Surgery, University Hospital of Cologne, Kerpener Strasse 62, 50937 Cologne, Germany

2 Department for Orthopedic and Trauma Surgery, St. Vinzenz Hospital, Merheimer Straße 221-223, 50733 Cologne, Germany

3 Department of Hand Surgery, Vulpius Klinik, Vulpiusstraße 29, 74906 Bad Rappenau, Germany

4 Department of Orthopedic Surgery, Yangpu Hospital, Tongji University School of Medicine, 450 Tengyue Road, 200090 Shanghai, People's Republic of China 